\title{
Interreality: The Experiential Use of Technology in the Treatment of Obe- sity
}

\author{
Riva G. ${ }^{1,2,}$, Wiederhold B.K. ${ }^{1,3}$, Mantovani F. ${ }^{4}$ and Gaggioli A., \\ ${ }^{1}$ ICE-NET Lab., Università Cattolica del Sacro Cuore, Milan, Italy \\ ${ }^{2}$ ATN-P Lab., Istituto Auxologico Italiano, Milan, Italy \\ ${ }^{3}$ Virtual Reality Medical Institute, Bruxelles, Belgium \\ ${ }^{4}$ Department of Epistemology and Hermeneutics of Education, University of Milan-Bicocca, Milan, Italy
}

\begin{abstract}
For many of us, obesity is the outcome of an energy imbalance: more energy input than expenditure. However, our waistlines are growing in spite of the huge amount of diets and fat-free/low-calorie products available to cope with this issue. Even when we are able to reduce our waistlines, maintaining the new size is very difficult: in the year after the end of a nutritional and/or behavioral treatment obese persons typically regain from $30 \%$ to $50 \%$ of their initial losses. A possible strategy for improving the treatment of obesity is the use of advanced information technologies. In the past, different technologies (internet, virtual reality, mobile phones) have shown promising effects in producing a healthy lifestyle in obese patients. Here we suggest that a new technological paradigm - Interreality - that integrates assessment and treatment within a hybrid experiential environment - including both virtual and real worlds - has the potential to improve the clinical outcome of obesity treatments. The potential advantages offered by this approach are: (a) an extended sense of presence: Interreality uses advanced simulations (virtual experiences) to transform health guidelines and provisions in experiences; (b) an extended sense of community: Interreality uses virtual communities to provide users with targeted - but also anonymous, if required - social support in both real and virtual worlds; (c) real-time feedback between physical and virtual worlds: Interreality uses bio and activity sensors and devices (smartphones) both to track in real time the behavior/health status of the user, and to provide targeted suggestions and guidelines. This paper describes in detail the different technologies involved in the Interreality vision. In order to illustrate the concept of Interreality in practice, a clinical scenario is also presented and discussed: Daniela, a 35-year-old fast-food worker with obesity problems.
\end{abstract}

Keywords: Interreality, obesity, virtual reality, biosensors, virtual communities, smartphones, experiential therapies.

\section{INTRODUCTION}

For many of us, obesity is the outcome of an energy imbalance: more energy input than expenditure. However, our waistlines are growing in spite of the huge amount of diets and fat-free/low-calorie products available. In fact, clinical practice shows that there may be multiple factors in addition to energy surplus involved in the aetiology of obesity $[1,2]$ : genetic, cultural, socioeconomic, psychological, situational, metabolic and physiological.

Furthermore, the situation is worsening rapidly. As recently noted by Flegal and colleagues [3] the prevalence of obesity for American adults aged 20 to 74 years increased by 7.9 percent for men and by 8.9 percent for women between 1976-1980 and 1988-1994. It increased subsequently by 7.1 percent for men and by 8.1 percent for women between 1988-1994 and 1999-2000 reaching 32.2\% among adult men and $35.5 \%$ among adult women in 2007-2008. The situation is similar in other countries. In Italy almost half of men and

*Address correspondence to this author at the Dipartimento di Psicologia, Università Cattolica del Sacro Cuore, Largo Gemelli 1, 20123 Milan, Italy; Tel: +39-02-619112726; Fax: +39-02-619112892;

E-mail: auxo.psylab@auxologico.it about 1 in 3 women are overweight or obese. Furthermore, between 2001 and 2008 the age-standardized prevalence of overweight (obesity) increased by $1.4 \%(1.9 \%)$ in men and by $0.4 \%(0.5 \%)$ in women [4]. Similar data can be found for Greece [5], Switzerland [6], and Brazil [7].

Why is obesity so difficult to treat? A possible answer is the following: obesity is not a homogenous disorder. In other words, clinical practice should always follow an individualized patient-oriented approach [8]. Indeed, clinical practice should aim to induce significant change in both behavior and actual weight, with long-term support to maintain therapeutic gains. As underlined by Brownell and Stunkard [9] "If a person loses to ideal weight and maintains it, eats a healthy diet, increases activity, and makes changes in psychological domains, all is well. However this is the perfect outcome and perfection is rare." (p.508).

A typical behavioral treatment can produce a $8 \%-10 \%$ body weight reduction over the course of 20-24 weekly sessions [10]. However, at the end of the treatment participants gradually regain weight [11]: in the year after the end of the treatment obese persons typically regain from $30 \%$ to $50 \%$ of their initial weight loss [12]. The situation is even worse in the long term and for morbid obesity [13]. 
As noted by Jeffery and colleagues [12]: "Fresh ideas are needed to push the field forward" (p.5). A possible strategy for improving the actual treatment of obesity is the use of advanced information technologies. In the past different technologies (internet, virtual reality, mobile phones) have shown promising effects in producing a healthy lifestyle in obese patients. Here we suggest that a new technological paradigm - Interreality - may improve the clinical outcome of obesity.

\section{ADVANCED TECHNOLOGY IN OBESITY TREATMENT: FROM VIRTUAL REALITY TO IN- TERREALITY}

The recent convergence between technology and medicine [14] is offering new methods and tools for behavioral healthcare [15-19]. Between them, an emerging trend is the use of virtual reality (VR) to improve the existing cognitive behavioral protocols for different psychological disorders, especially anxiety disorders [20-27]. Using this approach, therapists can provide in-office, systematic, controlled exposure therapy (virtual reality exposure - VRE) to anxious patients, mitigating many of the complications of the in vivo exposure [28]. Moreover, the specific characteristics of the VR experience provide patients with a safe setting where they can exist and experience life [29, 30], thereby allowing them them to be "emotionally present" inside the virtual environment [31-34]. VR can also be described as an advanced imaginal system: an experiential form of imagery that is as effective as reality in inducing emotional responses [35-37]). A recent meta-analysis [38] of VRE trials confirmed that in vivo treatment was not significantly more effective than VRE. In fact, there was a small effect size favoring VRE over in vivo conditions.

The use of VR has also been utilized in the treatment of obesity as part of Experiential Cognitive Therapy (ECT). Developed by Giuseppe Riva and his group, inside both the IVT2010 Italian Government-funded project and the VEPSY Updated European funded project, ECT is a relatively shortterm, patient-oriented approach that focuses on individual discovery. As in the case of Cognitive Behavioral Therapy (CBT), ECT uses a combination of cognitive and behavioral procedures to help the patient identify and change the maintaining mechanisms. However ECT differs from CBT in the following ways:

- Its use of VR: $10 \mathrm{VR}$ sessions are part of the standard protocol. The different virtual scenes are included in an open source virtual environment - NeuroVR [34, $39,40]$ - that can be freely downloaded from the NeuroVR web site: http://www.neurovr.org. Using this software the therapist may also customize each environment by adding significant cues (images, objects, and video) related to the story of the patient. As noted by Glantz et al. [41] "One reason it is so difficult to get people to update their assumptions is that change often requires a prior step - recognizing the distinction between an assumption and a perception. Until revealed to be fallacious, assumptions constitute the world; they seem like perceptions, and as long as they do, they are resistant to change." (p. 96). Using the 'sense of presence' induced by VR, it is easier for the therapist to create realistic experiences which demonstrate to patients that the 'reality' which they perceive - e.g., body image distortion - is in fact is a result of his/her mind. Once this has been understood, individual maladaptive assumptions can then be challenged more easily.

- Its focus on empowerment. Critical goals of ECT include empowering the patient to decide on a realistic target weight range, to monitor eating behaviors and tomanage frustration related to weight fluctuations. However, unlike the CBT approach, ECT holds that these skills are part of a broader dimension, defined as "control self-efficacy" [42]: "the individual's ability to control the addictive behavior in a variety of provocative situations" (p. 303). Following this approach, the main strategy to raise self-efficacy is "empowerment", the process of helping people feel a sense of control over their lives. VR has the right features to support empowerment (enhancing the capacity of individuals), since it is a special, sheltered setting where patients can start to explore and act without feeling threatened.

- Its focus on the negative emotions related to the body, a major reason patients want to lose weight: The VR environments are also used to induce a controlled sensory rearrangement that facilitates an update of the allocentric representation of the body [30, 43]. To reach this goal, ECT follows a specific protocol (for a detailed description see Thompson and colleagues [44], p. 322-325) based on the one defined by Cash $[45,46]$ for overcoming body image problems.

Experiential Cognitive Therapy has been tested in different case studies and controlled trials [47-52] and in general provides better results in the follow-up than competing approaches, including both nutritional [53] and cognitive behavioral therapy [54]. However, there is still room for improvement: only $47 \%$ of the treated sample was able to maintain or improve the weight reduction achieved after the treatment in a 12-month follow-up. Specifically, there are two major issues underlined by clinicians using ECT:

- ECT tries to change behaviors and cognitions at a more general level, rather than limiting changes to actual situations or the 'real' context in which they happen;

- The ECT protocol is not customized to the peculiar characteristics of the patient.

On one hand, in the ECT protocol VR is a distinct realm, separate from the emotions and behaviors experienced by the patient in the real world: the behavior of the patient in VR has no direct effect on real life experience; nor are the emotions and problems experienced by the patient in the real world directly addressed in the VR exposure.

On the other hand, ECT is a manualized approach that allows for a limited amount of customization to be made according to the specific characteristics of a given patient. As noted by Havik and Vanden Bros [55] "in the ordinary clinical setting, the most pressing immediate problem of real patients in psychotherapy may change from week to week, and the clinician must assess and address each new problem as it is presented, as well as attempt to relate it to the overall agreed-upon goals for the patient's psychotherapy." (pp.265266). 
In this paper we suggest that a further advancement of the treatment of obesity by VR can be offered by a new technological paradigm, Interreality: a hybrid, closed-loop empowering experience bridging physical and virtual worlds [56, 57]. The main feature of Interreality is a twofold link between the virtual and the real world: (a) behavior in the physical world influences the experience in the virtual one; (b) behavior in the virtual world influences the experience in the real one $[58,59]$.

The different technologies that are involved in the Interreality vision and its clinical rationale will be addressed and presented in the next paragraph.

\section{THE INTERREALITY PARADIGM: CLINICAL RATIONALE AND TECHNOLOGY}

\subsection{The Clinical Rationale}

According to the World Health Organization [60] and the American National Institute of Health [61] obesity treatment is based around the following goals:

- To minimize or reduce health risks in obese individuals through weight reduction.

- To prevent further weight gain.

- To initiate weight reduction when appropriate.

- To support the adoption of a healthy lifestyle and improve quality of life.

- To improve body image dissatisfaction and selfefficacy.

- To encourage appropriate follow-up for the purpose of maintaining a healthy or reduced weight.
Here we suggest that it is possible to improve the longterm treatment of obesity using a new paradigm for e-health - "Interreality" - that integrates contextualized assessment and treatment within a hybrid environment, bridging the physical and virtual world [56] (see Fig. 1).

Our claim is that bridging virtual experiences (fully controlled by the therapist, used to learn healthy behaviors and coping skills, and to improve body image) with real experiences (the therapist can identify critical situations and assess clinical change) - using advanced technologies (virtual worlds, advanced sensors and smartphones) is a feasible way to address the complexity of this disturbance.

Although classical behavioral and cognitive-behavioral approaches focus on directly modifying the content of dysfunctional behaviors and thoughts through a rational and deliberate process, Interreality focuses on modifying the patient's relationship with his/her behavior and thinking through more contextualized experiential processes.

On one hand, the patient is continuously assessed in the virtual and real worlds by tracking their behavioral and emotional status in the context of challenging tasks (customization of the therapy according to the characteristics of the patient). On the other hand, feedback is continuously provided to improve the patient's skills through a conditioned association between performance and execution of assigned tasks (improvement of self efficacy). In sum, from the clinical viewpoint, the Interreality paradigm may offer the following innovations to current obesity protocols:

- Objective and quantitative assessment of symptoms using biosensors and behavioral analysis: monitoring of the patient's behavior and both his general and

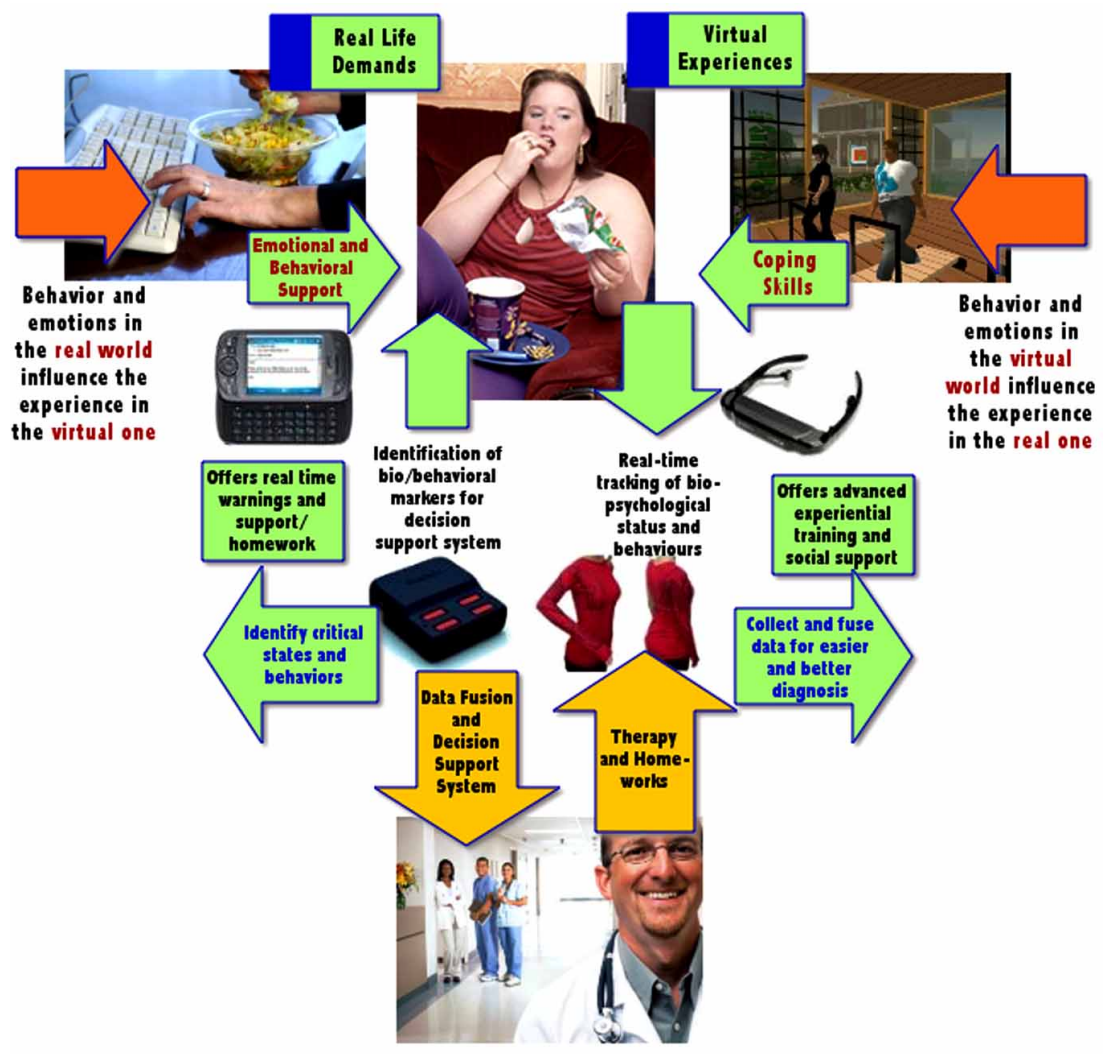

Fig. (1). The Interreality approach to obesity. 
psychological status, early detection of symptoms and timely activation of feedback in a closed-loop approach;

- Decision support for treatment planning through data fusion and detection algorithms: monitoring of patient's responses to treatment, management of the treatment and the provision of support for clinicians in making therapeutic decisions.

- Provision of warnings and motivating feedback to improve compliance and long-term outcome: the sense of "presence" allowed by this approach affords the opportunity to deliver behavioral, emotional and physiological self-regulation training in an entertaining and motivating fashion.

In conclusion, the Interreality approach provides a twofold feedback activity (Fig. 2):

- Behavior in the physical world influences experiences in the virtual world:

- For example, if I do not exercise during the day, my avatar will become fatter and the content of the virtual experience will increase its focus on improving motivation to perform physical exercise.

- Behavior in the virtual world influences experiences in the real world:

○ For example, if my emotional management skills in the virtual world have been poor, the decision support system may increase possible prompts or 'warnings' in real life and will provide additional homework assignments.

\subsection{The Technology}

From the technological viewpoint Interreality is based on the devices/platform described below:
- 3D Individual and/or shared virtual worlds: They allow controlled exposure, objective assessment, and the provision of motivating feedback.

- Personal digital assistants and/or mobile phones (from the virtual world to the real one). They allow objective assessment, the provision of warnings prompts and motivating feedback).

- Personal bio-monitoring system (from the real world to the virtual one). It allows: objective and quantitative assessment, decision support for treatment.

These devices are integrated around two subsystems: the Clinical Platform (inpatient treatment, fully controlled by the therapist) and the Personal Mobile Platform (real world support, available to the patient and connected to the therapist) that allow the:

a. Monitoring of the patient's behavior and his/her general and psychological status, early detection of symptoms and timely activation of feedback in a closed loop approach;

b. Monitoring of the response of the patient to treatment, management of the treatment and support for therapists in making therapeutic decisions.

Below we will detail the main features of the different technologies involved in these two platforms.

\subsubsection{The Virtual Worlds}

The virtual world component of the obesity protocol is composed of various 3D individual and/or shared virtual worlds organized around three different but interconnected islands/areas: the Learning Island, the Community Island and the Experience Island (Fig. 3).

1. The Learning Island: The goal of the Learning Island is to use motivation provided by the virtual worlds to

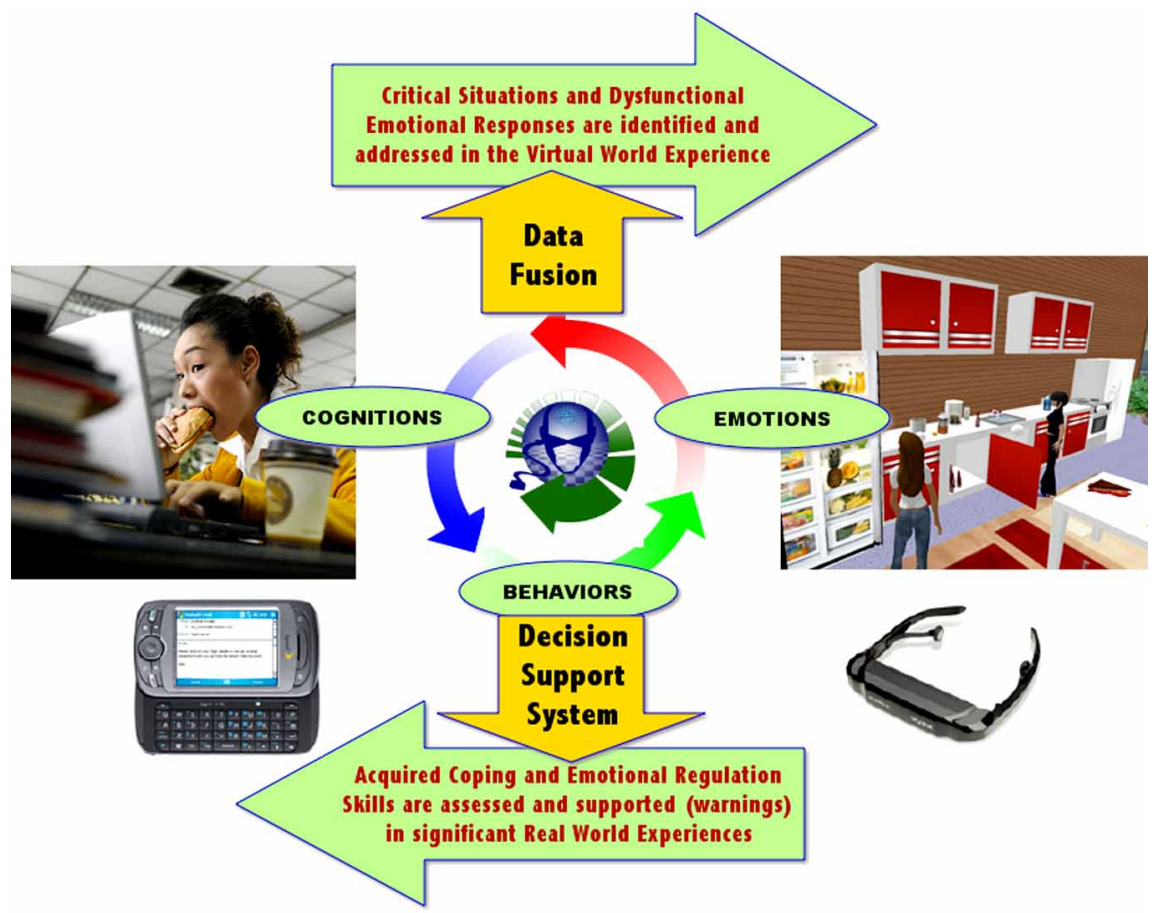

Fig. (2). The feedback provided to the patient by the Interreality approach. 
teach users about how to improve their eating and living habits. The Learning Island is organized around different learning areas both without and with teachers (classes).

In this island the user:

a. Learns what obesity is and its multi-factorial etiology;

b. Learns how to manage daily food choices and activities;

c. Learns workouts that target key muscle groups;

d. Samples hundreds of delicious recipes and meal ideas.

e. Learns to become aware of and modify unhelpful thoughts and maladaptive thinking about food;

f. Learns stress-relieving exercises (e.g., relaxation training or diaphragmatic breathing) should food be used as an emotional management tool;

g. Receives the information needed to succeed, with daily tips and expert ideas.

2. The Community Island: The goal of the Community Island is to use the strength of virtual communities to provide real-life insights aimed at improving eating and living habits. The Community Island is organized around different zones in which users discuss and share experiences between themselves with or without the supervision of an expert (physician, psychologist, nutritionist, etc.). In this island the user:

a. Enjoys support and guidance;

b. Learns successful and unsuccessful examples of problem-focused and emotion-focused coping strategies;

c. Learns how to make wise choices, eat healthy and enjoy food and exercise.

d. Benefits from the exchange of practical experiences and tips from other patients.

1. The Experience Island: The goal of the Experience Island is to use the feeling of presence provided by the virtual experience to practice both eating/emotional/ relational management and general decision-making

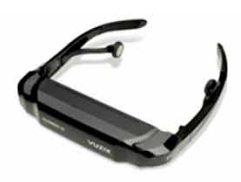

3D Individual and

Shared Virtual Worlds

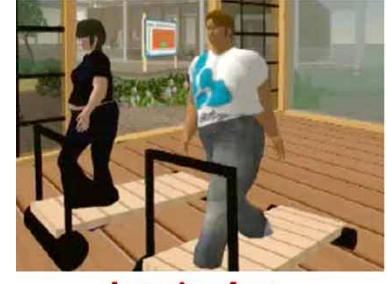

Learning Area:

To learn what obesity is and how to cope with it

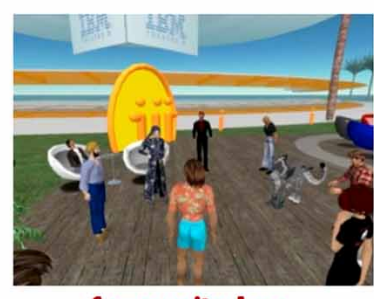

Community Area:

To meet other patients with

the same problems and problem-solving skills. The island includes different zones (presenting critical situations related to the maintaining/relapse mechanisms (Mall, Supermarket, Pub, Restaurant, Swimming Pool, Beach, Gymnasium, etc.) and two body image comparison areas. Each of these environments is experienced under supervision only. In these areas the user:

a. Is helped to develop specific strategies for avoiding and/or coping with critical situations. After the experience the coach explores the patient's understanding of what happened in the virtual experience and their specific reactions - emotional and behavioral - to the different situations experienced. If needed, some new strategies for coping with the situations are presented and discussed.

\subsubsection{The Real World Interface: A Smartphone}

In Interreality, a patient's activity in the virtual world has a direct link with his/her life through a mobile phone/digital assistant. This link is at three levels:

1. Follow-up (warnings and/or feedback): it is possible to assess/improve the outcome of the virtual experience through the smartphone, eventually also using the information coming from the bio and activity sensors.

- Example: if the real world outcome is poor after receiving a real time warning, the user will experience again the same virtual environment. If it is good, the user will receive real time motivating feedback and will be able to share his/her experience with other users.

2. Training/Homework: thanks to the advanced graphic/ communication capabilities now available on smartphones, they can be used as training/simulation devices to facilitate the real-world transfer of knowledge acquired in the virtual world.

- Example: The nutritional guidelines learned in the virtual world can be experienced in the real life context before or during a visit to the supermarket.

Fig. (3). The virtual experiences used by the Interreality approach.

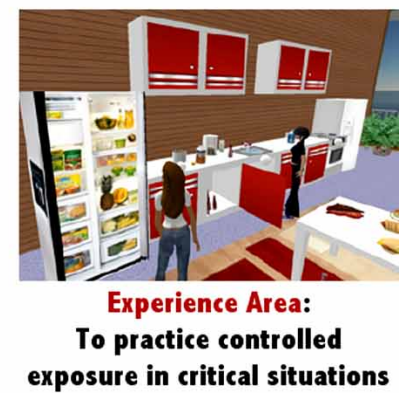


3. Community: the social links created in the virtual world can be continued in the real world even without revealing the real identity of the user.

- Example: I can SMS a virtual friend in my own real context to ask for support.

\subsubsection{The Personal Bio-Monitoring System: Behavioral and Physiological Sensors}

In Interreality the dynamic behavioral profile of the user (contextualized behaviors and body dynamics) and the physiological response of the user to events (analysis of biosensors' data) is done through a Personal Bio-monitoring System (PBS), consisting of independent wearable bands for the examination of the physiological and behavioral signs.

The PBS allows full-body motion tracking through a 3D wearable motion analysis platform. The PBS integrates biosensors for the transduction of heart rate variability (HRV), electro dermal response (EDR), peripheral skin temperature, as well as EEG data. The PBS will wirelessly integrate stateof-the-art miniature inertial sensors, wireless communication solutions and bio-electrodes, as well as conductive elastromer-based paths directly screen-printed on each single band for the electrical connections. GPS data for location-tracking will be obtained from the smartphone.

The full PBS system will be used in the therapist's office only. To improve acceptability, the patient will use a bluetooth wearable sensor only (HRV, EDR, skin temperature).

\section{INTERREALITY IN PRACTICE: A CLINICAL SCENARIO}

To present and discuss the clinical value of the Interreality paradigm, we will use a clinical scenario: Daniela, a 35 year old fast-food worker with obesity problems.

\subsection{The Clinical Scenario}

Daniela was 35 years old (Fig. 4), and she worked in a fast-food restaurant near the train station of her town. She was single and had struggled with her weight most of her life. Since adolescence, she had tried many different diets and weight-loss methods, but without results. Even worse, she gained more weight after each dieting attempt.

Her weight was $115 \mathrm{kgs}$ (BMI: 43.8) and the excess body fat was impacting her health: she was at risk for Type II diabetes, cardiovascular disease, hypertension and stroke. For this reason her physician suggested to her that she enter a weight-loss treatment program.

\subsection{The Clinical Protocol}

At admission, Daniela presented as anxious and somewhat sad. After a short assessment interview and paper-andpencil self-report questionnaires, she was diagnosed with mild depression and moderate anxiety.

After the assessment phase the therapist explained to Daniela the Interreality approach (see Fig. 5) and protocol (4 to 6 months of weekly sessions), and asked her to use the Interreality system.

The therapist showed Daniela how to wear biosensors to monitor her physiological parameters. Then, the therapist put the non-invasive sensors on Daniela and explained how to use them, beginning the education process.

After this short training phase, the therapist introduced Daniela to her first individual virtual experience: the psychologist's office. This represents the start and the end of each virtual experience, and it has the important function of outlining the boundaries of the VR session. It is a neutral and reassuring place, which allows continuity in the phases of the individual session: face-to-face, VR and face-to-face. In the

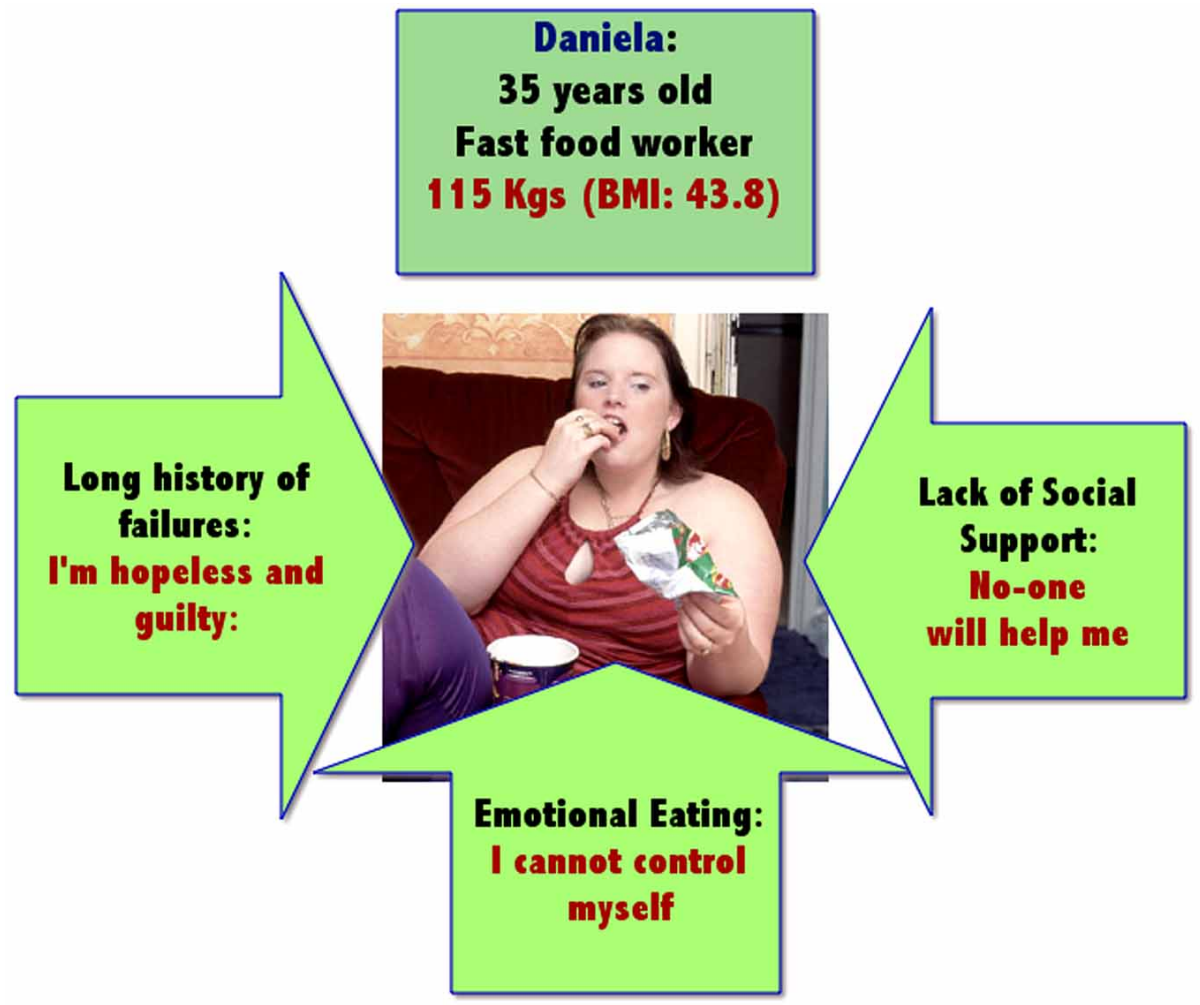

Fig. (4). Daniela - A clinical scenario. 


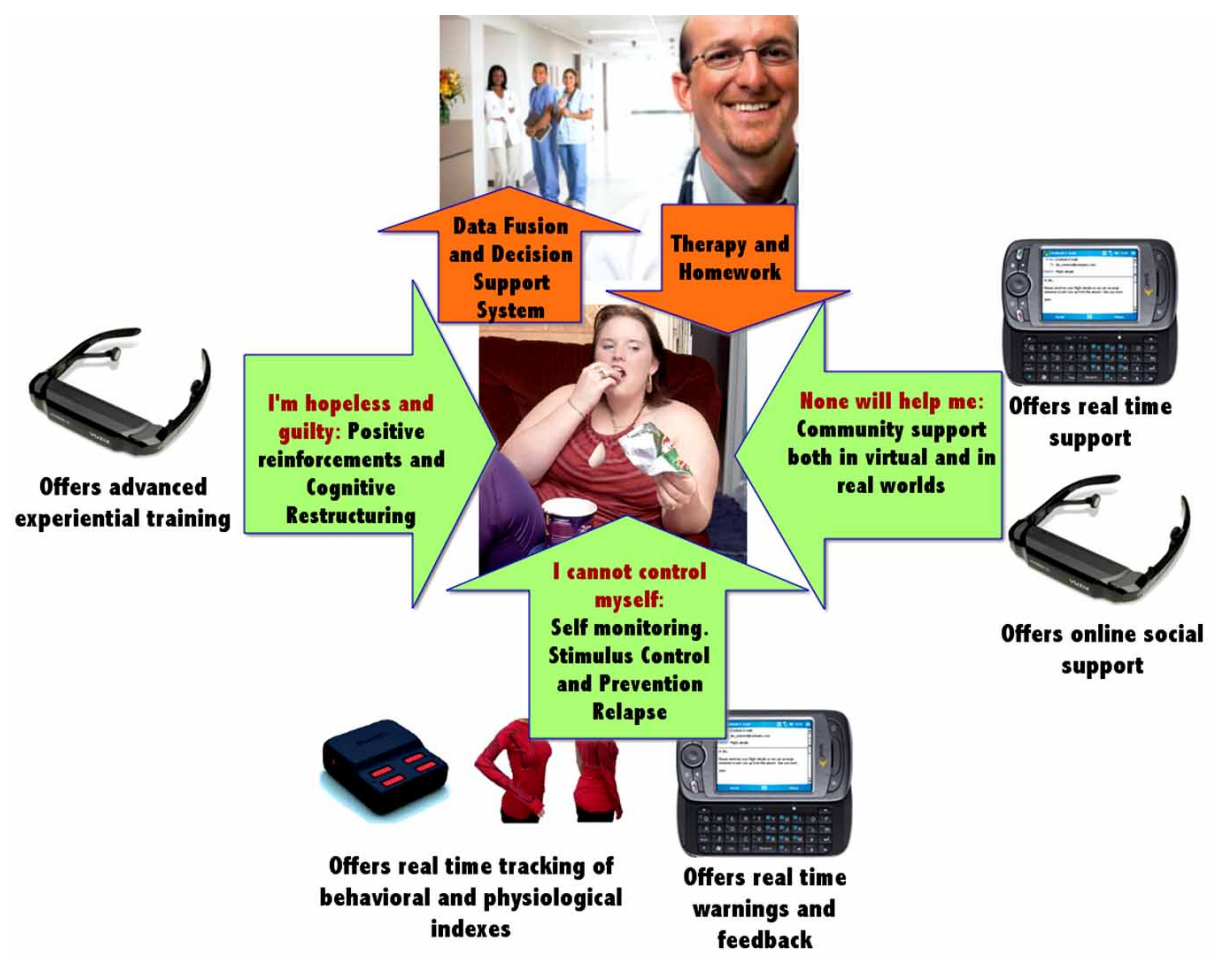

Fig. (5). The support provided by the Interreality approach to Daniela.

psychologist's office there are the following objects: a writing desk with two comfortable chairs, a bookshelf and furnishings that make the environment more comfortable and hospitable (pictures, carpets, lamps, green plants, etc.).

After experiencing the psychologist's office Daniela moved to one of the virtual worlds - the Experience Island where she entered the virtual balance room. Daniela weighed herself on the virtual scales in the middle of the room. When Daniela stood on the scales, her weight(which had previously been typed in) appeared on the display.

The therapist used this experience to explore any symptoms of anxiety related to the experience of weighing and to assess Daniela's concerns about eating, body shape and weight.

The interview during the virtual experience revealed a long history of increasing guilt and shame that had its focus in a growing disgust for her body. To counter it she had occasionally used drastic weight control measures in the past, but was unable to maintain the weight loss. The data fusion system, integrating all the biosensors' data, allowed the therapist to assess in real time how the virtual situation was affecting Daniela's physiological responses.

At the end of the clinical session, the therapist provided homework for Daniela. She was asked to use her smartphone to keep a daily log of food consumed and exercise. Specifically, Daniela was asked to input the food or the exercise in the smartphone immediately afterwards. At the same time the biosensors tracked her physiological responses. The aim was to understand what she had eaten and determine possible risk situations that may lead to negative behaviors. Using this data, both Daniela and the therapist learned, for example, at what time she usually ate, that she ate when stressed, or in the company of specific friends, etc.
The data reported by the smartphone was also used by the therapist to schedule the type and content of the feedback provided by the Decision Support System (DSS). Specifically it provided stimulus control by suggesting contextualized behaviors: restricting the number of places where eating was permitted, not missing meals, allowing eating only after specific activities etc.

In the following phase of the treatment, Daniela was invited to experience a new virtual world, the Learning Island, where she entered a Virtual Kitchen. This virtual room was a quite spacious environment with windows, an equipped kitchen (refrigerator, oven, gas-ring, sink, mixer, toaster, etc.) and a table with four chairs.

In the kitchen Daniela interacted with the presented objects: she opened the cupboards, the refrigerator, the freezer and the oven. Moreover, she was allowed to choose and "eat" any of the available food. This experience provided Daniela with information to help plan a healthy diet while managing calorie intake. Furthermore, after "eating" the different foods she was able to immediately see the impact of the choice on her avatar (it became thinner or fatter). She was then allowed to reverse her choice and learn which nutrients should be limited and which nutrients should be consumed in adequate amounts. During the experience, when the avatar became fatter she reported feeling guilty, ashamed, and even more uncomfortable in her body.

To address this strong emotional response, in a latter session the therapist asked Daniela to enter a new world in the Experience Island: the swimming pool. When Daniela entered this environment she found herself in front of a swimming pool surrounded by deck chairs, towels on the floor, and people sunbathing on the lawn and on the edge of the swimming pool. Some people were swimming. 
With the help of her therapist and of the data collected by the biosensors, Daniela began to recognize her own pattern of using eating to regulate emotions and also the negative emotions related to her body. In this process, Daniela was helped to identify and consciously experience her emotions so that she could choose appropriate distress tolerance skills to manage them. For example, in another world of the Experience Island - the relaxation area, a green valley with a lake in the middle - she learned some simple relaxation procedures. She was delighted at the emotional control this virtual experience allowed her to feel, and the relief of having tools to rely on during emotionally difficult moments rather than defaulting to eating behaviours.

Daniela also met virtually in the Learning Island with her dietician to assess her level of physical fitness and activity. Together, they explored the physical activities she enjoyed in the past or might enjoy if she gave herself permission to try. Based upon the results of this assessment, and with some coaching, Daniela began to exercise three times per week for 60 minutes.

Daniela was then invited to enter her first group virtual experience, the Community Island (under therapist supervision) - where she had the opportunity to share and discuss her experience with other patients who suffer from the same problems.

However, in some cases Daniela experienced new critical situations - i.e., her boss wanted her to do something that exceeded her available time and resources - that triggered emotional eating behaviors. When these situations occurred, the Decisions Support System provided her with some suggestions about dealing with negative emotions (i.e. instructions to relax) and guidelines to avoid seeing the lapse as a sign that she was a total failure leading to increased shame.

Daniela was scheduled to see the therapist the next day. During the session the therapist asked her if the suggestions provided by the Decision Support System were helpful. Daniela's answer was positive: the Decision Support System helped her to reframe her thoughts more positively. Further, it helped to remind her about the relaxation techniques that she had previously learned. Then, the therapist compared the information provided by Daniela with the data collected by the Decision Support System. This analysis allowed the therapist to identify critical issues - Daniela's difficulty in negotiating properly with her boss - and to define the next phases of the treatment.

In the following sessions, Daniela reported to the therapist that although her initial treatment goal was weight loss, this broader empowerment approach was necessary for her. She also reported that meeting other people in the Community Island helped her to find much-needed support and to discover new strategies to manage her negative emotions. For instance Rosa, a 42-year-old nurse with a 38 BMI, suggested to her that she participate more in fun activities to decrease stress and shame about her appearance. Paola, a 51year-old teacher with a 39 BMI told her to focus on small accomplishments, rather than just big ones. Following these suggestions Daniela learned to reframe her thoughts, with less self-condemnation.
Finally, having full-time support through the smartphone made Daniela more confident. She began to recognize lapses-i.e., eating out of emotion rather than hunger-and redirected herself to use the learned skills during these times.

At the end of the treatment Daniela had taken a pivotal step: she was no longer measuring the rate or amount of weight loss, but drawing empowerment from understanding the dynamics that had kept her bound in a yo-yo diet cycle most of her life. Daniela also understood that she could accept her body as it was and celebrate its changes in relation to her more balanced lifestyle and enjoyment of food.

\subsection{Challenges and Cost Effectiveness}

In a recent review, Riva [15] identified four major issues that may limit the use of the proposed approach:

- the lack of standardisation of virtual reality hardware and software and the limited possibility of tailoring virtual environments to the specific requirements of the clinical or the experimental setting;

- the low availability of standardised protocols that can be shared by the community of researchers;

- the high costs (up to $€ 200,000$ US\$) required for designing and testing it;

- expensive technical support or continual maintenance are often required.

Further, lack of ongoing training and technical support can discourage professionals from utilising the proposed approach with a significant waste of time and technology.

Finally, only a small number of clinical studies related to the use of technology in the treatment of obesity to date have made cost-effectiveness calculations. Costs for the Interreality approach include installation and maintenance of both fixed and mobile equipment, ongoing technical support, documentation requirements, accommodation and staff training.

On the other hand, the costs of overweight and obesity are huge. A recent study estimated that the yearly costs in UK for these disturbances are about $£ 3.2$ billion (US\$ 4 billion). The direct treatment costs of obesity include GP consultations, ordinary admissions, day cases, outpatient attendances and prescriptions. The indirect cost of obesity includes treating the consequences of this disturbance: stroke, CHD, hypertensive disease and diabetes mellitus.

Obesity has also a big impact on life expectancy: Craig and Tseng [62] suggest that, for a 55-year old man, an additional weight of $2.5 \mathrm{BMI}$ is associated with the loss of at least 2.15 years of life.

As underlined by Simpson [63] the evaluation of costs encountered or saved through the use of the Interreality approach must balance technological, training and support costs with factors such as reduced treatment costs, a longer lifetime horizon, improved family and work commitments, costs incurred through time and expense of travel.

Furthermore, some new studies suggest that when managed and organised well, the use of Interreality technologies (virtual reality and mobile phones) may lead to cost savings 
and improved outcome for both patients and health services (e.g. $[64,16])$.

\section{CONCLUSIONS}

The use of new technologies (Internet, virtual reality, mobile phones), and in particular virtual reality (VR), is not new in the treatment of obesity: VR is part of Experiential Cognitive Therapy (ECT), a relatively short-term, patientoriented approach that focuses on individual discovery. In this approach VR is used as an "empowering environment", a special, sheltered setting where patients can start to explore and act without feeling threatened [33, 65]. Nothing the patient fears can "really" happen to them in VR. With such assurance, they can freely explore, experiment, feel, live and experience feelings and/or thoughts.

ECT has been tested in different case studies and controlled trials [47-52] and in general it provides better results in the follow-up than competing approaches including both nutritional [53] and the cognitive behavioral therapy [54].

However, as discussed before, until now this approach has not addressed the following issues:

- VR is a new and distinct realm, separate from the emotions and behaviors experienced by the patient in the real world;

- The protocol has not been customized to the peculiar characteristics of the patient.

To overcome the above limitations, here we suggested a new e-health paradigm - "Interreality" - that integrates assessment and treatment within a hybrid experiential environment including both virtual and real worlds $[58,59]$.

Classical behavioral and cognitive-behavioral approaches to obesity focus on directly modifying the content of dysfunctional behaviors and thoughts through a rational and deliberate process $[66,67]$. In Interreality this happens within more contextualized experiential processes.

Specifically, the clinical use of Interreality is based on a closed-loop concept that involves the use of technology for assessing, adjusting and/or modulating the behaviors and emotions of the patient in both real and virtual worlds. On one hand, the patient is continuously assessed in the virtual and real worlds by tracking their behavioral and emotional status in the context of challenging tasks (customization of the therapy according to the characteristics of the patient). On the other hand, feedback is continuously provided to improve the skills of the patient through a conditioned association between performance and execution of assigned tasks (improvement of self efficacy).

To discuss and evaluate the clinical use of the proposed approach we presented and detailed a possible clinical scenario: Daniela, a 35-year-old fast-food worker with obesity problems.

From this clinical case, we suggested that the potential advantages offered to obesity treatments by the interreallity approach are:

- An extended sense of presence: Interreality uses advanced simulations (virtual experiences) to transform health guidelines and provisions in experience. In Interreality the patients do not receive abstract information but live meaningful experiences;

- An extended sense of community: Interreality uses hybrid social interaction and dynamics of group sessions to provide each user with targeted - but also anonymous, if required - social support in both physical and virtual worlds.

- Real-time feedback between physical and virtual worlds: Interreality uses bio and activity sensors and devices (smartphones) both to track in real-time the behavior and the health status of the user and to provide targeted suggestions and guidelines.

Obviously, any new paradigm requires a lot of effort and time to be assessed and properly used. Without a real clinical trial with obese patients, the Interreality paradigm will remain an interesting, but untested concept.

Only a small number of clinical studies related to the use of technology in the treatment of obesity to date have made cost-effectiveness calculations. Costs for the Interreality approach include installation and maintenance of both fixed and mobile equipment, ongoing technical support, documentation requirements, accommodation and staff training.

On the other hand, direct and indirect costs of overweight and obesity are huge; and some new studies suggest that when managed and organised well, the use of Interreality technologies may lead to cost savings and improved outcome for both patients and health services (e.g. [16, 64]).

In conclusion, despite the lack of clinical data, we suggest the Interreality paradigm may represent a valid opportunity for improving the long-term outcome of obesity treatments. Our hope is that the present paper will stimulate a discussion within the clinical and research community about the potential, the advantages and the possible risks that bridging physical and virtual world offers to cybertherapy applications.

\section{ACKNOWLEDGEMENTS}

The work for preparing this paper was partially supported by the European funded project "Interstress" - Interreality in the management and treatment of stress-related disorders (FP7-247685 - http://www.interstress.eu). The authors wish also to thank the anonymous reviewers for their suggestions and comments.

\section{REFERENCES}

[1] Muruganathan A. Obesity and weight management in primary care. J Indian Med Assoc 2009; 107(7): 441-5.

[2] Apovian CM. The causes, prevalence, and treatment of obesity revisited in 2009: what have we learned so far? Am J Clin Nutr 2010; 91(1): 277S-9S.

[3] Flegal KM, Carroll MD, Ogden CL, Curtin LR. Prevalence and trends in obesity among US adults, 1999-2008. JAMA 2010; 303(3): 235-41.

[4] Micciolo R, Di Francesco V, Fantin F, et al. Prevalence of overweight and obesity in italy (2001-2008): is there a rising obesity epidemic? Ann Epidemiol 2010; 20(4): 258-64.

[5] Tambalis KD, Panagiotakos DB, Kavouras SA, et al. Eleven-year prevalence trends of obesity in Greek children: first evidence that prevalence of obesity is leveling off. Obesity 2010; 18(1): 161-6.

[6] Faeh D, Bopp M. Increase in the prevalence of obesity in Switzerland 1982-2007: birth cohort analysis puts recent slowdown into perspective. Obesity 2010; 18(3): 644-6. 
[7] de Andrade RG, Pereira RA, Sichieri R. Ten-year increase in the prevalence of obesity and reduction in fat intake in Brazilian women aged 35 years and older. J Epidemiol Commun Health 2010; 64(3): 252-4.

[8] Blaine BE. Obesity, binge eating, and psychological distress: The moderating role of self-concept disturbance. Curr Psychiat Rev 2009; 5(3): 175-81.

[9] Brownell KD, Stunkard AJ. Goals of obesity treatment. In: Fairburn CG, Brownell KD, Eds. Eating Disorders and Obesity: A comprehensive handbook. New York: The Guilford Press 2002; p. 507-11.

[10] Perri MG, Fuller PR. Success and failure in the treatment of obesity: Where do we go from here? Med Exerc Nutr Health 1995; 4: 255-72.

[11] Hankey CR. Session 3 (Joint with the British Dietetic Association): Management of obesity: Weight-loss interventions in the treatment of obesity. Proc Nutr Soc 2010; 69(1): 34-8.

[12] Jeffery RW, Drewnowski A, Epstein LH, et al. Long-term maintenance of weight loss: Current status. Health Psych 2000; 19 (Suppl.): 5-16.

[13] Blackburn GL, Wollner S, Heymsfield SB. Lifestyle interventions for the treatment of class III obesity: a primary target for nutrition medicine in the obesity epidemic. Am J Clin Nutr 2010; 91(1): 289S-92S.

[14] Riva G. Ambient intelligence in health care. Cyberpsychol Behav 2003; 6(3): 295-300.

[15] Riva G. Virtual reality: an experiential tool for clinical psychology. Br J Guid Counsel 2009; 37(3): 337-45.

[16] Preziosa A, Grassi A, Gaggioli A, Riva G. Therapeutic applications of the mobile phone. Br J Guid Counsel 2009; 37(3): 313-25.

[17] Gorini A, Gaggioli A, Riva G. Virtual worlds, real healing. Science 2007; 318(5856): 1549 .

[18] Gorini A, Gaggioli A, Vigna C, Riva G. A second life for eHealth: prospects for the use of 3-D virtual worlds in clinical psychology. J Med Internet Res 2008; 10(3): e21.

[19] Wiederhold BK, Wiederhold MD. The future of Cybertherapy: Improved options with advanced technologies. Stud Health Technol Inform 2004; 99: 263-70.

[20] Riva G, Molinari E, Vincelli F. Interaction and presence in the clinical relationship: virtual reality (VR) as communicative medium between patient and therapist. IEEE Trans Inf Tech Biomed 2002; 6(3): 198-205.

[21] Riva G. Virtual reality in psychotherapy: review. Cyberpsychol Behav 2005; 8(3): 220-30; discussion 31-40.

[22] Moore K, Wiederhold BK, Wiederhold MD, Riva G. Panic and agoraphobia in a virtual world. Cyberpsychol Behav 2002; 5(3): 197-202.

[23] Parsons TD, Rizzo AA. Affective outcomes of virtual reality exposure therapy for anxiety and specific phobias: A meta-analysis. J Behav Ther Exp Psychiatry 2008; 39(3): 250-61.

[24] Saunders T, Driskell JE, Johnston JH, Salas E. The effect of stress inoculation training on anxiety and performance. J Occup Health Psychol 1996; 1(2): 170-86.

[25] Riva G, Algeri D, Pallavicini F, Repetto C, Gorini A, Gaggioli A. The use of advanced technologies in the treatment of psychological stress. J Cyberther Rehab 2010; 2(2): 169-71.

[26] Pallavicini F, Algeri D, Repetto C, Gorini A, Riva G. Biofeedback, VR and Mobile Phones in the treatment of Generalized Anxiety Disorders: A phase-2 controlled trial. J Cyberther Rehab 2009; 2(4): 315-28.

[27] Wender R, Hoffman H, Hunner H, Seibel E, Patterson D, Sharar S. Interactivity influences the magnitude of virtual reality analgesia. $\mathrm{J}$ Cyberther Rehab 2009; 2(1): 27-34.

[28] Botella C, Quero S, Banos RM, Perpina C, Garcia Palacios A, Riva G. Virtual reality and psychotherapy. Stud Health Technol Inform 2004; 99: 37-54

[29] Baños RM, Botella C, Alcañiz M, Liaño V, Guerrero B, Rey B. Immersion and Emotion: Their impact on the sense of presence. CyberPsychol Behav 2004; 7(6): 734-41.

[30] Riva G. From virtual to real body: Virtual reality as embodied technology. J Cyberther Rehab 2008; 1(1): 7-22.

[31] Hoffman HG, Richards T, Coda B, Richards A, Sharar SR. The illusion of presence in immersive virtual reality during an fMRI brain scan. Cyberpsychol Behav 2003; 6(2): 127-31.
[32] Riva G. From technology to communication: Psycho-social issues in developing virtual environments. J Vis Lang Computing 1999; 10: 87-97.

[33] Riva G, Gaggioli A, Gorini A, et al. Virtual reality as empowering environment for personal change: the contribution of the applied technology for neuro-psychology laboratory. Anuario Psicol 2009; 40(2): 182-205.

[34] Riva G, Gaggioli A, Villani D, et al. NeuroVR: an open source virtual reality platform for clinical psychology and behavioral neurosciences. Stud Health Technol Inform 2007; 125: 394-9.

[35] North MM, North SM, Coble JR. Virtual reality therapy for fear of flying. Am J Psychiatry 1997; 154(1): 130.

[36] Vincelli F. From imagination to virtual reality: the future of clinical psychology. Cyberpsychol Behav 1999; 2(3): 241-8.

[37] Vincelli F, Molinari E, Riva G. Virtual reality as clinical tool: immersion and three-dimensionality in the relationship between patient and therapist. Stud Health Technol Inform 2001; 81: 551-3.

[38] Powers MB, Emmelkamp PM. Virtual reality exposure therapy for anxiety disorders: A meta-analysis. J Anx Dis 2008; 22(3): 561-9.

[39] Algeri D, Carelli L, Gaggioli A, Riva G. A Free tool for motor rehabilitation: NeuroVR 1.5 with CamSpace. Stud Health Technol Inform 2009; 144: 99-101.

[40] Carelli L, Morganti F, Poletti B, et al. A NeuroVR based tool for cognitive assessment and rehabilitation of post-stroke patients: two case studies. Stud Health Technol Inform 2009; 144: 243-7.

[41] Glantz K, Durlach NI, Barnett RC, Aviles WA. Virtual reality (VR) and psychotherapy: Opportunities and challenges. Presence 1997; 6(1): 87-105.

[42] DiClemente CC. Self-efficacy and the addictive behaviors. J Soc Clin Psych 1986; 4: 302-15.

[43] Riva G. Neuroscience and Eating Disorders: The role of the medial temporal lobe. Nature Precedings 2010; doi: 10101/mpre 2010.4235 .

[44] Thompson JK, Heinberg LJ, Altabe M, Tantleff-Dunn S. Exacting beauty: Theory, assessment and treatment of body image disturbance. Washington DC: American Psychological Association 1999.

[45] Cash TF. The body image workbook: an eight-step program for learning to like your looks. Oakland, CA: New Harbinger 1997.

[46] Cash TF. The treatment of body image disturbances. In: Thompson JK, editor. Body image, eating disorders and obesity. Washington, DC: APA - American Psychological Association1996; p. 83-107.

[47] Riva G. The virtual environment for body-image modification (VEBIM): Development and preliminary evaluation. Presence 1997; 6(1): 106-17.

[48] Riva G. Modifications of body image induced by virtual reality. Percept Mot Skills 1998; 86: 163-70.

[49] Riva G. Virtual environment for body-image modification: Virtual reality system for the treatment of body image disturbances. Comp Hum Behav 1998; 14(3): 477-90.

[50] Riva G, Bacchetta M, Baruffi M, Rinaldi S, Vincelli F, Molinari E. Virtual reality-based experiential cognitive treatment of obesity and binge-eating disorders. Clin Psych Psychother 2000; 7(3): 209-19.

[51] Riva G, Bacchetta M, Baruffi M, Molinari E. Virtual reality-based multidimensional therapy for the treatment of body image disturbances in obesity: a controlled study. Cyberpsychol Behav 2001; 4(4): 511-26.

[52] Riva G, Bacchetta M, Cesa G, et al. Is severe obesity a form of addiction? Rationale, clinical approach, and controlled clinical trial. Cyberpsychol Behav 2006; 9(4): 457-79.

[53] Brownell KD. The LEARN manual for weight control. Philadelphia: University of Pennsylvania Press; 1985.

[54] Cooper Z, Fairburn CG, Hawker DM. Cognitive-Behavioral Treatment of Obesity: A Clinician's guide. New York: Guilford Publications, Inc 2003.

[55] Havik OE, Vanden Bos GR. Limitations of manualized psychotherapy for everyday clinical practice. Clin Psych: Sci Pract 2006; 3(3): 264-7.

[56] Riva G. Interreality: A New Paradigm for E-health. Stud Health Technol Inform 2009; 144: 3-7.

[57] van Kokswijk J. Hum@n, Telecoms \& Internet as Interface to Interreality. Hoogwoud, The Netherlands: Bergboek 2003. 
[58] Riva G, Raspelli S, Pallavicini F, et al. Interreality in the management of psychological stress: a clinical scenario. Stud Health Technol Inform 2010; 154: 20-5.

[59] Riva G, Raspelli S, Algeri D, et al. Interreality in practice: bridging virtual and real worlds in the treatment of posttraumatic stress disorders. Cyberpsychol Behav Soc Netw 2010; 13(1): 55-65.

[60] WHO. Redefining obesity and its treatment. Manila, Phillippines: World Health Organization (International Association for the Study of Obesity, International Obesity Task Force) 2000

[61] NIH. Obesity: the practical guide. Identification, evaluation, and treatment of overweight and obesity in adults. Bethesda, MD: National Institutes of Health, NHLBI Obesity Education Initiative 2000.

[62] Craig BM, Tseng DS. Cost-effectiveness of gastric bypass for severe obesity. Am J Med 2002; 113(6): 491-8.
[63] Simpson S. Psychotherapy via videoconferencing: a review. Br J Guid Counsel 2009; (37) : 3 .

[64] Wood DP, Murphy J, McLay R, et al. Cost effectiveness of virtual reality graded exposure therapy with physiological monitoring for the treatment of combat related post traumatic stress disorder. Stud Health Technol Inform 2009; 144: 223-9.

[65] Botella C, GarcÌa-Palacios A, Villa $\mathrm{H}$, et al. Virtual reality exposure in the treatment of panic disorder and agoraphobia: A controlled study. Clin Psych Psychother 2007; 14(3): 164-75.

[66] Fairburn CG. Cognitive-behavioral treatment for bulimia. In: Garner DM, Garnfinkel PE, editors. Handbook of psychotherapy for anorexia and bulimia. New York: Guilford Press 1985 p. 16092.

[67] Wilson GT, Fairburn CG. Cognitive Treatments for Eating Disorders. J Consult Clin Psych 1993; 61(2): 261-9.

(C) Riva et al.; Licensee Bentham Open.

This is an open access article licensed under the terms of the Creative Commons Attribution Non-Commercial License (http://creativecommons.org/licenses/by-nc/3.0/) which permits unrestricted, non-commercial use, distribution and reproduction in any medium, provided the work is properly cited. 